\title{
3D Reconstruction From A Single Image Based On Shape Evolution
}

\author{
Qian Ma \\ Wuhan University of Science and Technology City College, Wuhan, 470083, China \\ email: icey_mq@163.com
}

Keywords: SFS; Improved Arrival Time Method; 3D Segmentation; Shape Evolution

\begin{abstract}
D reconstruction from a single image is a challenging problem. In this paper, we present an improved method for 3D reconstruction. We use improved arrival time method to solve the Hamilton-Jacobi equation which is obtained from the method of shape from shading (SFS). The concave-convex ambiguity problems occurred like with other SFS methods, so we find out the boundary of the concave-convex regions according to 3D Segmentation based on Gaussian curvature, then reconstruct the surface use the shape evolution method based on energy constraint in order to obtain the accuracy 3D shape.
\end{abstract}

\section{Introduction}

The shape from shading is a method to recover the 3D shape of an object by using the gray information of a given image, it is a difficult problem in the field of computer vision. The problem was originally proposed by Horn[1] in 1970, but it has been proved that the SFS is an ill posed problem because of the convex-concave ambiguities. Oliensis[2] and Kozera[3] has discussed the existence and uniqueness of the solution for the SFS problem. According to this, Dupuis and Oliensis[4] presented a monotone convergence algorithm to recovered the surface of objects. Subsequently Oliensis[5] further relaxed the assumptions, analyzed the situation which uniquely identified in most areas of the image and polysemy in local area. In addition, there are SFS methods based on Neural Networks[6-10], based on Green function[11] and so on.

In this paper, we transform the gray equation of the image into the corresponding static Hamilton-Jacobi equation in the solution of SFS problem, then use the Level set method with the improved arrival time method to obtain the viscosity solution. The final result will be of the convex-concave ambiguities, so we find boundary line based on Gaussian curvature for 3D segmentation, then reconstruct the curved surface to get the accurate $3 \mathrm{D}$ model by the shape evolution based on energy constraint.

\section{Solving The SFS Based On Improved Arrival Time Method}

General methods for the SFS are based on the following assumptions: (1)The light source is an infinite point light source which is equivalent to parallel light; (2)The reflection model is a Lambert diffuse reflection model; (3)Imaging relation is parallel orthogonal projection; (4)The object surface reflectance is 1 . Based on the above assumptions, the direction of the light source is $(0,0,1)$, the unit normal vector of the surface is $\left(z_{x}, z_{y}, 1\right)$. The relationship between the image radiance $E(x, y)$ and the corresponding point on the surface is:

$$
E(x, y)=\frac{1}{\sqrt{z_{x}^{2}+z_{y}^{2}+1}}
$$

So we can calculate the height of the point $(x, y)$ by the intensity of each point of the image, so as to restore the shape of the original surface. There is only one equation, but there are two unknown parameters. Therefore, other constraints must be added to solve the equation.

Evolutionary method follow the reference point which has the definite shape or height, along the path of the evolution of the image in order to gradually calculate the height of the other points in the image, so as to obtain the surface height of the whole object. Oliensis[12], Bruekstein[13], Rouy[14] 
and Osher[15] et al illustrated the SFS is a kind of solving power system from the view of dynamic point, which can be regarded as a Hamilton system problem, solving the problem is to solve a Hamilton-Jacobi equation:

$$
H=E(x, y)-\frac{1}{\sqrt{z_{x}^{2}+z_{y}^{2}+1}}
$$

This is a first order nonlinear partial differential equation, if given initial conditions, the equation is a Cauchy problem, if given boundary conditions, the equation constitutes a Dirichlet problem. There are many methods to solve this problem, such as characteristic strips method, viscosity solution method, minimum downhill method and level set method.

We rewritten the equation(2) based on the level set method and $\nabla z(x, y)=\left(z_{x}, z_{y}\right)$ as follow:

$$
\|\nabla z(x, y)\|=\sqrt{\frac{1}{E(x, y)^{2}}-1}
$$

Through put the brightness function into equation(3), height function can be obtained by solving the above equation. Then we will improve the arrive time method to carry out the solution. Rewritten equation(3) as:

$$
\|\nabla z(x, y)\|=f(x, y)
$$

It is a static Hamilton-Jacobi equation, the discrete form of $\|\nabla z\|$ is:

$$
\begin{aligned}
\left\{\left[\max \left(D_{x}^{-} z_{i j}, 0\right)\right]^{2}+\left[\min \left(D_{x}^{+} z_{i j}, 0\right)\right]^{2}+\left[\max \left(D_{y}^{-} z_{i j}, 0\right)\right]^{2}+\left[\min \left(D_{y}^{+} z_{i j}, 0\right)\right]^{2}\right\}^{1 / 2} & =f_{i j} \\
i & =1, \ldots, N, j=1, \ldots M
\end{aligned}
$$

where $D_{x}^{-} z_{i j}, D_{x}^{+} z_{i j}, D_{y}^{-} z_{i j}, D_{y}^{+} z_{i j}$ respectively for the first order forward and backward difference in $x$ and $y$ directions:

$$
D_{x}^{-} Z_{i j}=\frac{Z_{i, j}-z_{i-1, j}}{\Delta x}, D_{x}^{+} Z_{i j}=\frac{Z_{i+1, j}-z_{i, j}}{\Delta x}, D_{y}^{-} z_{i j}=\frac{z_{i, j}-z_{i, j-1}}{\Delta y}, D_{y}^{+} Z_{i j}=\frac{Z_{i, j+1}-Z_{i, j}}{\Delta y}
$$

According to the characteristics of the arrival time function we changed equation(5) to:

$$
\left\{\left[\max \left(D_{x}^{-} z_{i j},-D_{x}^{+} z_{i j}, 0\right)\right]^{2}+\left[\max \left(D_{y}^{-} z_{i j},-D_{y}^{+} z_{i j}, 0\right)\right]^{2}\right\}^{1 / 2}=f_{i j} \quad i=1, \ldots, N, j=1, \ldots M
$$

In order to improve the accuracy, we take the second difference to calculate $\|\nabla z\|$ in any of the following two situations: (1)The point in all directions and its distance from two points must be known; (2)In all directions, the arrival time value of two points away from the point is less than or equal to the one point away from it. Other cases are still using the first difference.

Two order forward difference and the backward difference formula are as follows:

$$
\begin{aligned}
& D_{x}^{-} z_{i j}=\frac{3 z_{i, j}-4 z_{i-1, j}+z_{i-2, j}}{2 \Delta x}, D_{y}^{-} z_{i j}=\frac{3 z_{i, j}-4 z_{i, j-1}+z_{i, j-2}}{2 \Delta y} \\
& D_{x}^{+} z_{i j}=-\frac{3 z_{i, j}-4 z_{i+1, j}+z_{i+2, j}}{2 \Delta x}, D_{y}^{+} z_{i j}=-\frac{3 z_{i, j}-4 z_{i, j+1}+z_{i, j+2}}{2 \Delta y}
\end{aligned}
$$

Substituted the above function in equation(6), we can have

$$
\begin{aligned}
& {\left[\max \left(\frac{3 z_{i, j}-4 z_{i-1, j}+z_{i-2, j}}{2 \Delta x}, \frac{3 z_{i, j}-4 z_{i+1, j}+z_{i+2, j}}{2 \Delta x}, 0\right)\right]^{2}+} \\
& \quad\left[\max \left(\frac{3 z_{i, j}-4 z_{i, j-1}+z_{i, j-2}}{2 \Delta y}, \frac{3 z_{i, j}-4 z_{i, j+1}+z_{i, j+2}}{2 \Delta y}, 0\right)\right]^{2}=f_{i j}^{2}
\end{aligned}
$$

Rewritten it as follow:

$$
\begin{aligned}
& {\left[\max \left(\frac{3 z_{i, j}-\min \left(4 z_{i-1, j}-z_{i-2, j}, 4 z_{i+1, j}-z_{i+2, j}\right)}{2 \Delta x}, 0\right)\right]^{2}+} \\
& \quad\left[\max \left(\frac{3 z_{i, j}-\min \left(4 z_{i, j-1}-z_{i, j-2}, 4 z_{i, j+1}-z_{i, j+2}\right)}{2 \Delta y}, 0\right)\right]^{2}=f_{i j}^{2}
\end{aligned}
$$


Let $Z=z_{i, j}, Z_{1}=\min \left(4 z_{i-1, j}-z_{i-2, j}, 4 z_{i+1, j}-z_{i+2, j}\right), Z_{2}=\min \left(4 z_{i, j-1}-z_{i, j-2}, 4 z_{i, j+1}-z_{i, j+2}\right)$

Then

$$
\left[\max \left(\frac{3 Z-Z_{1}}{2 \Delta x}, 0\right)\right]^{2}+\left[\max \left(\frac{3 Z-Z_{2}}{2 \Delta y}, 0\right)\right]^{2}=f_{i j}^{2}
$$

To solve this equation and obtain the 3D reconstruction model, the experimental results are shown in Figure 1.

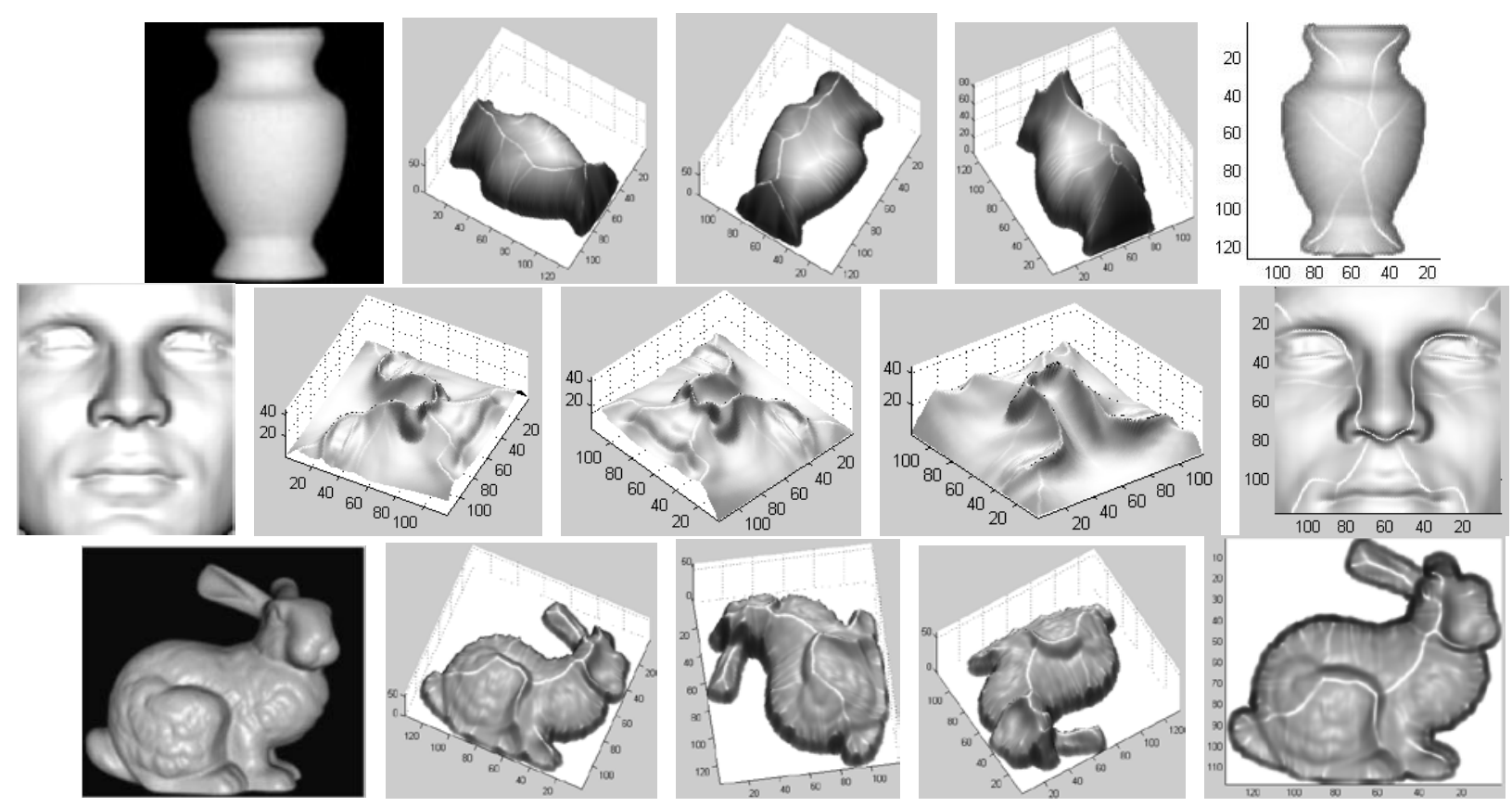

Fig. 1 The 3D reconstruction models based on improved arrival time method

\section{Find Boundary Line Based On Gaussian Curvature For 3D Segmentation}

As we can see in the Figure 1, the integrity of the shape is relatively good, but it cannot avoid convex-concave ambiguities. So we need the subsequent processing in order to obtain accurate 3D models. So we first find out the boundary of the convex-concave regions use the method of 3D Segmentation based on Gaussian curvature.

We find out the points which have the minimum negative curvature by estimation the Gaussian curvature of each point, the boundaries are made of these points. First, calculate the Gaussian curvature of each point using the vertex position of the neighborhood, the flare angle of the adjacent triangle, and the area of the adjacent triangle. The calculation formula as follow:

$$
K(p)=\frac{3\left(2 \pi-\sum_{i=1}^{N} \vartheta_{i}\right)}{\sum_{i=1}^{N} A_{i}} \delta^{2}\left(p-p_{i}\right)
$$

Mark the points as the boundary points which's Gaussian curvature are bigger than the threshold, the others as the seed points. The choice of the threshold will affect the performance of the segmentation region growing. Then we delete the isolated points. The isolated point is the boundary point which has seed points around it, or the seed point which has boundary points around it. We change their mark to the same point as it is around. In this way, the isolated points are eliminated. To perform the iteration region growing method for each seed point, the area of the seed points are gradually increased, at the last the area are surrounded by the boundary. We put the points which have no mark to the certain area by its sign of the curvature and the distance of the neighborhoods. Finally, we get the determined boundaries and the dividing surfaces. The experimental results are shown in Figure 2. 

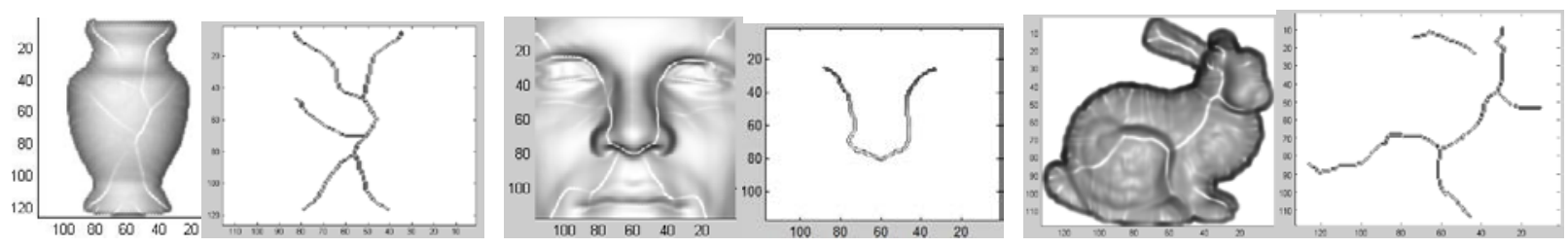

Fig. 2 The boundary lines

\section{Surface Evolution Based On Energy Constraint}

In this section, we will recover the concave into convex with the relative shape invariant. So we reconstruct the surface based on energy constraint. The internal energy of the surface depends on the nature of the surface itself. For curved F, its internal energy can be expressed as:

$$
E_{\text {int }}(F)=\int_{F}\left(\kappa_{1}^{2}+\kappa_{2}^{2}\right) d \omega
$$

where the $\kappa_{1}$ and $\kappa_{2}$ represent the first and second principal curvature. $d \omega$ represent the surface element. In order to simplify the calculation, the approximation is expressed as:

$$
E_{\text {int }}(F)=\int_{F}\left(F_{x x}^{2}+2 F_{x y}^{2}+F_{y y}^{2}\right) d x d y
$$

where $F_{x x}, F_{x y}, F_{y y}$ represent the second-order differential of $F$.

The essence of the external energy constraint is a constraint condition for the change of the surface shape. We construct additional term for the corresponding surface energy function, which can let the minimum energy surface formed by the deformation to meet the requirements of the constraints of the shape change. We take point restriction here. We give a point $P$ in the space, and pull the specified point $M$ which is on the surface to point $P$, then the surface shape changed correspondence. The corresponding external energy term is given as:

$$
E_{\text {ext }}(F)=c\|M-P\|^{2}
$$

where $c$ is the constant weight factor.

When the point $P$ on the deformed surface coincide with the specified point $P$, the external energy term get the minimum value. Usually, due to the influence of other energy terms in surface energy, the final minimum energy surface is not the least one. The greater the constant weight, the greater the influence of the point constraint energy term relative to the energy of the surface, and the surface more close to the point $P$.

When calculating the surface deformation, it is required that the boundary of the surface is bound to the original position of the surface, so that the surface can be kept unchanged before and after the deformation. So this is also used as the constraint condition to the external energy constraint, which is superimposed on the surface energy.

Therefore, the total energy of the surface $E$ is constructed from the internal energy and the external energy:

$$
E=E_{\text {ext }}+E_{\text {int }}
$$

We calculate the variation of total energy before and after the surface deformation. It can be considered that the total energy is the minimum when $\Delta E$ obtain the smallest value, and the surface $\bar{F}$ is what we ask for. So the problem of surface reconstruction is transformed into a non constrained optimization problem:

$$
\min \Delta E
$$

This unconstrained optimization problem can be solved by using conjugate gradient method. Using this method, we can obtain the correct 3D models. 


\section{Test results}

We have performed many experiments on different images. First we recovery the 3D shape from the image by SFS based on the improved arrival time method. We show the experimental results in Figure 1, Figure 2 and Figure 3. From Figure1, it is clear that the concave-convex ambiguity produced at some surfaces. Then we found the boundary of the concave-convex ambiguities using the method of 3D Segmentation based on Gaussian curvature, and the experimental results are shown in Figure 2. At last, we reconstructed the surface using the method of energy constraint, the experimental results are shown in Figure 3. Obviously, we can reconstructed the surface keeping the relative shape invariant find the boundary and raise the concave parts for a variety of different facial.
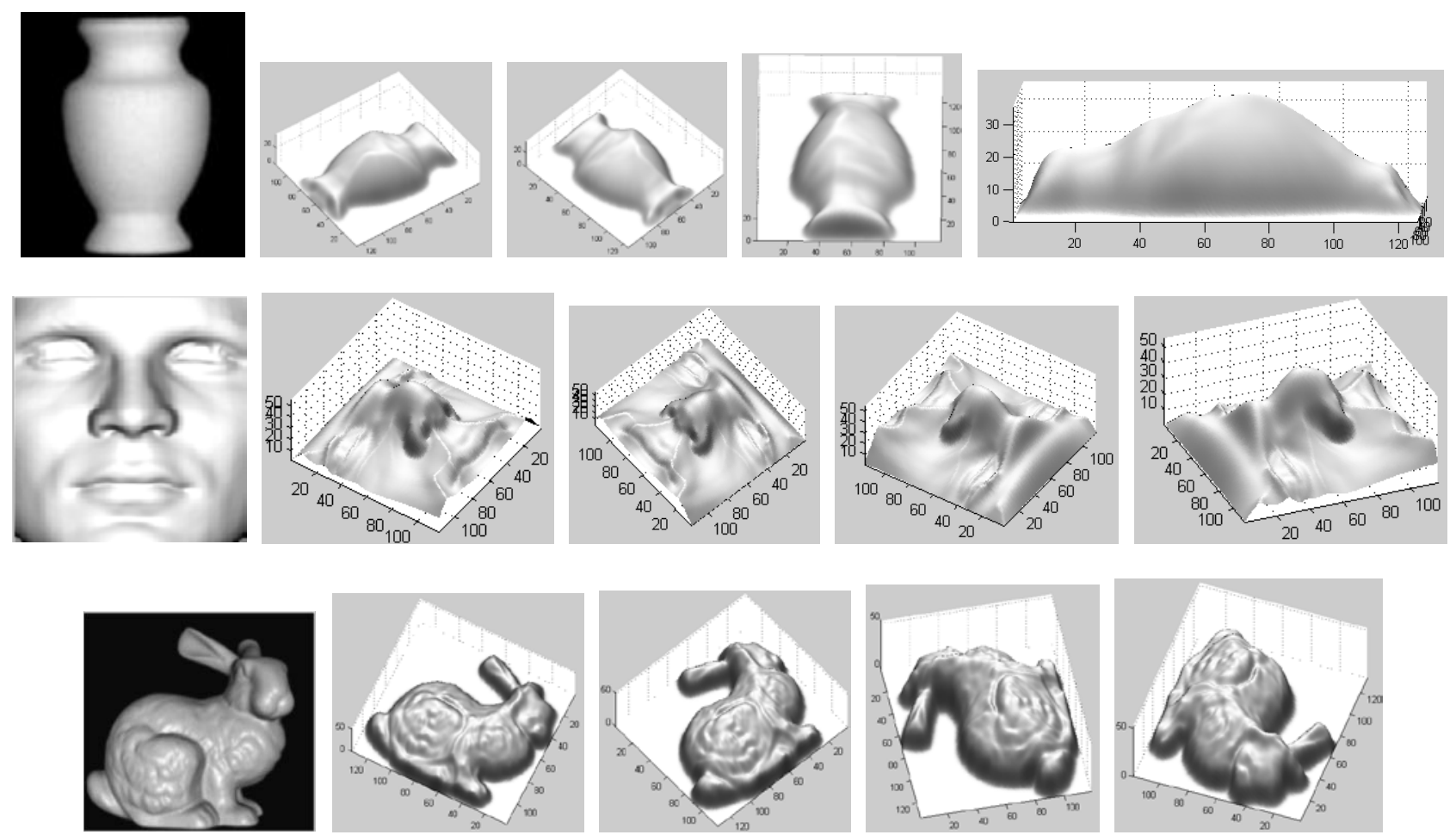

Fig. 3 The 3D models recovering from different images

\section{Conclusion}

In this paper, we have introduced a theoretical and computation framework to recover the 3D shape from a single frontal image. Experimental results have shown that our method is feasible and effective. Although the algorithm proposed in this paper has made some improvements, but still exists some shortage, there are some room for improvement, for example, the lighting environment is simple, it can be further study of other lighting environment, and the algorithm based on orthogonal projection is quite harsh hypothesis, because in the actual situation of perspective projection in more often.

\section{References}

[1] Horn B. K. P. Obtaining shape from shading information. Chapter 4 in The Psychology of Computer Vision, P.H.Winston(ed.), McGraw Hill, New York, 1975, p. 115-155.

[2] J. Oliensis. Uniqueness in Shape from Shading. International Journal of Computer Vision, 1991, 6(2):75 104.

[3] P. Dupuis, J. Oloensis. Shape from Shading: Provably Convergent Algorithms and Results. In: 
Computer Vision-ECCV, 1994. 259 268.

[4] R.Kozera. Uniqueness in Shape from Shading Revisited. Journal of Mathematical Imaging and Vision, 1997(7): 123 138.

[5] J.Oliensis. Shape from Shading as a Partially Well-Constrained problem. CVGIP: Image Understanding, 1991,54(2):163 183.

[6] G.-Q. Wei, G.Hirzinger. Parametric Shape-from-Shading by Radial Basis Functions.In: IEEE Transactions on Pattern Analysis and Machine Intelligence, 1997,19(4):353 365.

[7] T.-E.Kim, S.-H.Lee, et al. Shape Recovery of Hybrid Reflectance Surface using Neural Network. In: IEEE International Conference on Intelligent Processing Systems, 1997(3):416 419.

[8] J.Ben-Arie, D.Nandy. Aneuracwork approach for reconstructing surface shape from shading. In:IEEE International Conference on Intelligent Processing Systems, 1998(2):972 976.

[9] S.-Y.Cho, T.W.S.Chow. Shape Recovery from Shading by a New Neural-Based Reflectance Model. IEEE Transactions on Neural Networks, 1999, 10(6):1536 1541.

[10] M.G.-H. Mostafa, S.M. Yamany, A.A. Farag. Integrating Shape from Shading and Range Data Using Neural Networks. IEEE Computer Society Conference on Computer Vision and Pattern Recognition, 1999(1):15 20.

[11] J.R.A. Torreao. Shape from Shading and Intensity Gradient. XIII Brizilian Symposium on Computer Graphics and Image Processing, 2000. 7 14.

[12] Oliensis J. Shape from shading as a partially well-constrained problem[J]. CVGIP: IU., 1991, 54(2): 163-183.

[13] Bruckstein A M. On shape from shading[J]. CVGIP. , 1988,(44): 139-154.

[14] Rouy E, Tourin A. A viscosity solutions approach to shape-from-shading[J]. S IAM Journal of Numerical Analysis, 1992, 29(3): 867-884.

[15] Osher S . A level set formulation for the solution of the dirichlet problem for hamilton-jacobi equation [J ]. SIAMJ. Math. Anal. ,1993, 24(5): 1145-1152. 